\title{
An Idealized Modeling Study of the Midlatitude Variability of the Wind-Driven Meridional Overturning Circulation
}

\author{
Michael A. Spall ${ }^{\mathrm{a}}$ \\ ${ }^{a}$ Woods Hole Oceanographic Institution, Woods Hole, Massachusetts
}

(Manuscript received 23 December 2020, in final form 7 May 2021)

\begin{abstract}
The frequency and latitudinal dependence of the midlatitude wind-driven meridional overturning circulation (MOC) is studied using theory and linear and nonlinear applications of a quasigeostrophic numerical model. Wind forcing is varied either by changing the strength of the wind or by shifting the meridional location of the wind stress curl pattern. At forcing periods of less than the first-mode baroclinic Rossby wave basin crossing time scale, the linear response in the middepth and deep ocean is in phase and opposite to the Ekman transport. For forcing periods that are close to the Rossby wave basin crossing time scale, the upper and deep MOC are enhanced, and the middepth MOC becomes phase shifted, relative to the Ekman transport. At longer forcing periods the deep MOC weakens and the middepth MOC increases, but eventually for long enough forcing periods (decadal) the entire wind-driven MOC spins down. Nonlinearities and mesoscale eddies are found to be important in two ways. First, baroclinic instability causes the middepth MOC to weaken, lose correlation with the Ekman transport, and lose correlation with the MOC in the opposite gyre. Second, eddy thickness fluxes extend the MOC beyond the latitudes of direct wind forcing. These results are consistent with several recent studies describing the four-dimensional structure of the MOC in the North Atlantic Ocean.
\end{abstract}

SIGNIFICANCE STATEMENT: The purpose of this study is to better understand how large-scale winds at midlatitudes move water northward or southward, even in the deep ocean that is not in direct contact with the atmosphere. This is important because winds can shift where heat is stored and whether it might be released into the atmosphere. Our results provide a guide on what controls this motion and highlight the importance of large-scale ocean waves and smaller-scale ocean turbulence on the water movement and heat storage.

KEYWORDS: Eddies; Large-scale motions; Meridional overturning circulation; Ocean dynamics; Planetary waves

\section{Introduction}

The Atlantic meridional overturning circulation (AMOC) at midlatitudes represents a northward flow of warm, salty water in the upper ocean and a deeper southward flow of cooler, fresher waters carried by both the deep western boundary current and interior pathways. At $26.5^{\circ} \mathrm{N}$, the AMOC transports approximately $1.25 \times 10^{15} \mathrm{~W}$ of heat toward the north, which represents the dominant component of the oceanic meridional heat transport at this latitude (McCarthy et al. 2015; Roemmich and Wunsch 1985). AMOC transport changes are correlated with observed multidecadal variations of the largescale sea surface temperature distribution and surface heat fluxes (Latif et al. 2006; Knight et al. 2005) and so understanding what drives variability of AMOC and the related ocean heat content is central to understanding the ocean's role in climate variability.

Over $90 \%$ of the global heat gain over the past 60 years is stored in the ocean (Levitus et al. 2012; Abraham et al. 2013). Roughly $30 \%$ of that heat gain has occurred in the North Atlantic Ocean (Häkkinen et al. 2015). The warming has not been monotonic, however, as heat storage and heat exchange with the atmosphere show strong variability on seasonal to interannual and decadal time scales. Model studies clearly show that wind forcing is important for much of this observed

Corresponding author: Michael A. Spall, mspall@whoi.edu variability in heat content, especially so for the North Atlantic subtropical gyre and the eastern subpolar gyre (Lozier et al. 2008; Grist et al. 2010; Häkkinen et al. 2015; Williams et al. 2014; Evans et al. 2017; Pillar et al. 2016), and at mid- and low latitudes in the Pacific Ocean (Tandon et al. 2020). Huang (2015) termed such adiabatic, wind-driven oscillations of heat content as "Heaving Modes."

On very short time scales, the connection between winds and the overturning circulation stems from variations in sea surface height resulting from Ekman transport, which in turn drives a barotropic flow in the opposite direction (Bryan 1982; Jayne and Marotzke 2001). Because the ocean is stratified, this winddriven overturning results in a net meridional heat transport and temporary heat storage. Much of the overturning measured by the RAPID-MOCHA array at $26.5^{\circ} \mathrm{N}$ can be reproduced by simple wind-driven isopycnal models at both seasonal (Kanzow et al. 2010; Zhao and Johns 2014a; Yang 2015) and interannual time scales (Zhao and Johns 2014b; Pillar et al. 2016; Zou et al. 2019, 2020). Spall and Nieves (2020) found that midlatitude variability in winds could force a remote meridional overturning circulation (MOC) whose strength was strongly dependent on the forcing frequency and the baroclinic Rossby wave speed. While a direct connection between Ekman transport and the MOC is expected based on the barotropic response, these more recent studies find that at these lower frequencies the response is both baroclinic and nonlocal, indicating that more complex dynamics are at play. 
More complete wind- and buoyancy-forced basin and global scale models have also been used to assess the relative importance of wind and buoyancy forcing in MOC variability. Several recent studies (Biastoch et al. 2008; Yeager and Danabasoglu 2014; Polo et al. 2014; Pillar et al. 2016) have found buoyancy-forcing to be most important in decadal MOC variability but that wind forcing is dominant at shorter time scales. Grist et al. (2010) also found that, on interannual time scales, changes in ocean heat content in the North Atlantic were dominated by advective flux convergence, not air-sea exchange. Tandon et al. (2020) have found wind forcing dominates MOC variability in the Pacific Ocean on annual to interannual time scales, and that the associated meridional heat transport is the dominant component of the interannual variability in global meridional heat transport.

Several recent studies have begun to provide more detail on the vertical and meridional structure of the MOC variability on seasonal to decadal time scales. Frajka-Williams et al. (2016) found that the Ekman transport in the RAPID-MOCHA array was anticorrelated with the MOC in the Lower North Atlantic Deep Water but was uncorrelated with the Upper North Atlantic Deep Water, pointing to a deep overturning cell at time scales from seasonal to interannual. Isopycnal displacements on the western boundary support this variability in the MOC. A similar wind-driven deep overturning cell was found in the Pacific Ocean by Tandon et al. (2020). Zou et al. (2019) use an eddy-resolving model of the North Atlantic, an eddy-permitting global model, and an ocean reanalysis product to show that variability in the Lower North Atlantic Deep Water component of the MOC (approximately between $2000 \mathrm{~m}$ and the bottom) is correlated between the subpolar and subtropical gyres but the Upper North Atlantic Deep Water component of the MOC (depths between the surface and $2000 \mathrm{~m}$ ) is not. Bingham et al. (2007) find a mix of a meridionally coherent component of the MOC on interannual time scales but also stronger, high-frequency localized variability that results in a drop in the meridional coherence near $40^{\circ} \mathrm{N}$. This is consistent with Zou et al. (2020), who find two dominant modes of variability in the MOC. One mode is coherent at all latitudes between $25^{\circ}$ and $53^{\circ} \mathrm{N}$ and is associated with changes in the wind strength on interannual to decadal time scales. The second mode is anticorrelated across the subtropical and subpolar gyres and is associated with a meridional shift of the double gyre wind stress curl pattern on interannual time scales.

There are numerous observationally based studies that have identified correlations between MOC, oceanic heat content, and wind forcing. Williams et al. (2014) used reanalysis data to demonstrate that heat content variability in the North Atlantic dominates the secular warming trend over the past 60 years and is due primarily to heat flux convergence, not anomalous airsea heat fluxes. Note that this differs from analysis confined to the seasonal mixed layer, where local air-sea forcing can account for most of the variability in ocean heat content (Buckley et al. 2015). The subtropical gyre thermal anomalies were controlled by wind-induced changes to the Ekman heat convergence while in the subpolar gyre the thermal anomalies are of opposite sign and due to horizontal heat convergence by the MOC minus the Ekman transport. This is consistent with the findings of Häkkinen et al. (2015), who used reanalysis and observational datasets from the 1950s to 2012. Evans et al. (2017) used an ECCO ocean state estimate together with an Argo-based climatology to explicitly relate variability in ocean heat content in the North Atlantic subtropical gyre with variability in the MOC. They find that variations in the volume and heat budgets of the subtropical gyre are dominated by transport divergences within the gyre.

Dynamical understanding to date has been derived largely from sensitivity studies of either forward running numerical models or linearized adjoint models, both of which are typically run with realistic domains and forcing fields. Most proposed mechanisms involve westward-propagating baroclinic Rossby waves generated in the ocean interior or at the eastern boundary by changes in the wind stress curl, drawing on the fundamental study of transient adjustments to wind forcing by Anderson and Gill (1975). Previous idealized modeling studies (Kanzow et al. 2010; Zhao and Johns 2014a; Yang 2015; Huang 2015; Spall and Nieves 2020) have considered only the first baroclinic mode and linear dynamics. The present study uses theory and an eddy-resolving three-layer quasigeostrophic (QG) numerical model to address the midlatitude response to time-dependent winds with a focus on the influences of mesoscale eddies and higher baroclinic modes. A quasigeostrophic numerical model is introduced in section 2, followed in section 3 by an example of midlatitude variability in the MOC forced by oscillating winds. A quasigeostrophic, long-wave, analytic model of wind-driven MOC variability is presented in section 4. The numerical and analytical models are used in section 5 to understand how the MOC response (phase, amplitude, correlations) depends on the forcing frequency, pattern of wind variability, and presence of mesoscale eddies. A final summary is provided in section 6 .

\section{A quasigeostrophic model}

A quasigeostrophic model is used to study the variability of the meridional overturning circulation forced by timedependent winds. Quasigeostrophic models are not typically used to study the MOC. This is partly due to the fact that the MOC is fundamentally a buoyancy-forced circulation with important diapycnal transformations taking place near the surface and large-scale advection of these water masses away from their formation regions taking place at depth. This implies large deviations of isopycnals, which violates a basic assumption of the quasigeostrophic formulation. Another aspect of the global-scale MOC is that it spans a large range of latitudes and the equator, another problem for quasigeostrophic physics. However, the midlatitudes are far from both the highlatitude outcrop regions and the equator, and are known to be strongly wind forced, and so are suitable for quasigeostrophic physics. Because the QG equations do not explicitly solve for the ageostrophic motions the full MOC cannot be calculated directly from the horizontal velocity field. However, the MOC can be inferred from evolution of the perturbation density field, as discussed in detail below.

There are several advantages offered by QG physics. First, QG models can be configured as adiabatic, thus avoiding 
problems arising from artificial numerical diffusion that can be found in depth-coordinate, hybrid-coordinate, and sigmacoordinate primitive equation models. This allows for simple diagnostics and closure of water mass budgets. Second, QG models are computationally efficient, which allows for sufficient resolution to perform many century-long eddy resolving calculations. The QG model used for the present study employs a flux-limited second-order upwind advection scheme (CABARET), which results in well resolved, strong separated jets in wind-driven double gyre simulations (Karabasov et al. 2009). Third, the layered configuration makes for a simple interpretation of the vertical structure in terms of vertical modes.

The leading-order balances in the quasigeostrophic equations are geostrophic and hydrostatic:

$$
u=-\psi_{y}, \quad v=\psi_{x}, \quad \text { and } \quad b=f_{0} \psi_{z},
$$

where $f_{0}$ is the Coriolis parameter (constant over the domain), $u$ and $v$ are the zonal and meridional geostrophic velocities, $\psi$ is the velocity streamfunction, $b=-g \rho / \rho_{0}$ is the buoyancy, and subscripts indicate partial differentiation. Parameters $x, y, z$, and $t$ are respectively the zonal, meridional, vertical, and temporal coordinates, with $y=0$ at the southern boundary and $x=0$ at the western boundary.

The model solves the quasigeostrophic potential vorticity equation, which, in continuous form, is written as

$$
q_{t}+\psi_{x} q_{y}-\psi_{y} q_{x}=F+D, \quad q=\nabla^{2} \psi+\beta y+\left(\frac{f_{0}^{2}}{N^{2}} \psi_{z}\right)_{z},
$$

where $q$ is the potential vorticity, $\beta$ is the meridional gradient in planetary vorticity, $N$ is the Brunt-Väisälä frequency, $F$ represents forcing, and $D$ represents dissipation.

The model is discretized into three layers in the vertical direction. The governing equation and definition of potential vorticity for each layer are

$$
q_{k t}+\psi_{k x} q_{k y}-\psi_{k y} q_{k x}=F_{k}+D_{k} \quad \text { and } \quad q_{k}=\nabla^{2} \psi_{k}+\beta y+S_{k},
$$

where the subscript $k$ is the model layer and $S_{k}$ represents the stretching of planetary vorticity:

$$
\begin{aligned}
& S_{1}=\frac{f_{0}^{2}}{g_{12} H_{1}}\left(\psi_{2}-\psi_{1}\right), \\
& S_{2}=\frac{f_{0}^{2}}{g_{12} H_{2}}\left(\psi_{1}-\psi_{2}\right)+\frac{f_{0}^{2}}{g_{23} H_{2}}\left(\psi_{3}-\psi_{2}\right), \text { and } \\
& S_{3}=\frac{f_{0}^{2}}{g_{23} H_{3}}\left(\psi_{2}-\psi_{3}\right) .
\end{aligned}
$$

The reduced gravity between layers is defined as $g_{i k}=g\left(\rho_{i}-\rho_{k}\right) / \rho_{0}$, where $\rho_{0}$ is a reference density and $H_{k}$ is the mean thickness of each layer.

Dissipation is parameterized as a fourth-order term in each layer and a second-order term in the bottom layer, representing a bottom Ekman layer:

$$
D_{k}=\nu \nabla^{4} \psi_{k}-r \delta_{k 3} \nabla^{2} \psi_{k}
$$

$\delta$ is the Kronecker delta such that $\delta_{k i}=1$ for $k=i$ and is otherwise zero. For all calculations in the paper, $\nu=100 \mathrm{~m}^{2} \mathrm{~s}^{-1}$ and $r=4 \times 10^{-8} \mathrm{~s}^{-1}$. Partial-slip lateral boundary conditions are implemented through a Dirichlet boundary condition on all sidewalls for $\nabla^{2} \zeta_{k}$, where $\zeta_{k}=\nabla^{2} \psi_{k}$ is the relative vorticity. For example, at the eastern boundary

$$
\zeta_{k}(x=L)=\left.\psi_{k x x}\right|_{x=L}=\left.\alpha \psi_{k x}\right|_{x=L}
$$

and the inverse length scale $\alpha^{-1}=120 \mathrm{~km}$. This is intermediate to $\alpha^{-1}=\infty$ for free slip and $\alpha^{-1}=0$ for no slip (Karabasov et al. 2009).

The QG model is applied to the classic problem of a midlatitude wind-driven double gyre circulation. Essential information and parameter values are provided here, for a detailed description of the model and its numerical implementation the reader is referred to Karabasov et al. (2009). The model domain is a square with dimensions $L=5120 \mathrm{~km}$ and horizontal grid spacing of $10 \mathrm{~km}$. The domain is located at midlatitudes with $f_{0}=10^{-4} \mathrm{~s}^{-1}$ and $\beta=2 \times 10^{-11} \mathrm{~m}^{-1} \mathrm{~s}^{-1}$. The domain is $4000 \mathrm{~m}$ deep with a flat bottom and vertical sidewalls. The vertical stratification is represented by three layers with layer thicknesses $H_{1}=350 \mathrm{~m}, H_{2}=650 \mathrm{~m}, H_{3}=3000 \mathrm{~m}$. The reduced gravity between layers 1 and 2 is $g_{12}=0.0213 \mathrm{~m}^{2} \mathrm{~s}^{-1}$, and between layers 2 and 3 it is $g_{23}=0.0176 \mathrm{~m}^{2} \mathrm{~s}^{-1}$. This results in a first baroclinic deformation radius of $40 \mathrm{~km}$ and a second baroclinic deformation radius of $20 \mathrm{~km}$. The model is forced with a wind stress curl that is defined as

$$
\begin{aligned}
& F_{k}=-\delta_{k, 1} \frac{\pi \tau}{\rho_{0} L_{g} H_{1}} \sin \left[\pi\left(y-y_{0}\right) / L_{g}\right] \quad\left|y-y_{0}\right|<L_{g} \\
& F_{k}=0 \quad\left|y-y_{0}\right| \geq L_{g}
\end{aligned}
$$

$L_{g}=1536 \mathrm{~km}$ is the meridional extent of the subtropical and subpolar gyres. The wind stress will be varied in two ways. First, the amplitude will fluctuate sinusoidally in time with frequency $\omega$, with amplitude $\tau^{\prime}=0.4$, and will have a stationary distribution centered at the midlatitude of the domain $\left(y_{0}=\right.$ $0.5 L=2560 \mathrm{~km})$ :

$$
\tau=\tau_{0}\left(1+\tau^{\prime} \sin \omega t\right) .
$$

A second form of time dependence is introduced by keeping the magnitude of the wind stress constant $\left(\tau^{\prime}=0\right)$ and varying its meridional location as

$$
y_{0}=L\left(0.5+y^{\prime} \sin \omega t\right)
$$

with $y^{\prime}=0.1$. This results in a wind pattern that shifts north and south by $512 \mathrm{~km}$ with frequency $\omega$.

\section{An example of wind-driven variability}

The model was initialized at rest and run for a period of 120 years with $\tau_{0}=0.12 \mathrm{~N} \mathrm{~m}^{-2}, \tau^{\prime}=0$, and $y^{\prime}=0$. The mean transport streamfunction and layer thicknesses over the final 100 years are shown in Fig. 1. The mean circulation is dominated by a double gyre system separated by a sharp jet that penetrates several thousand kilometers into the basin. The 

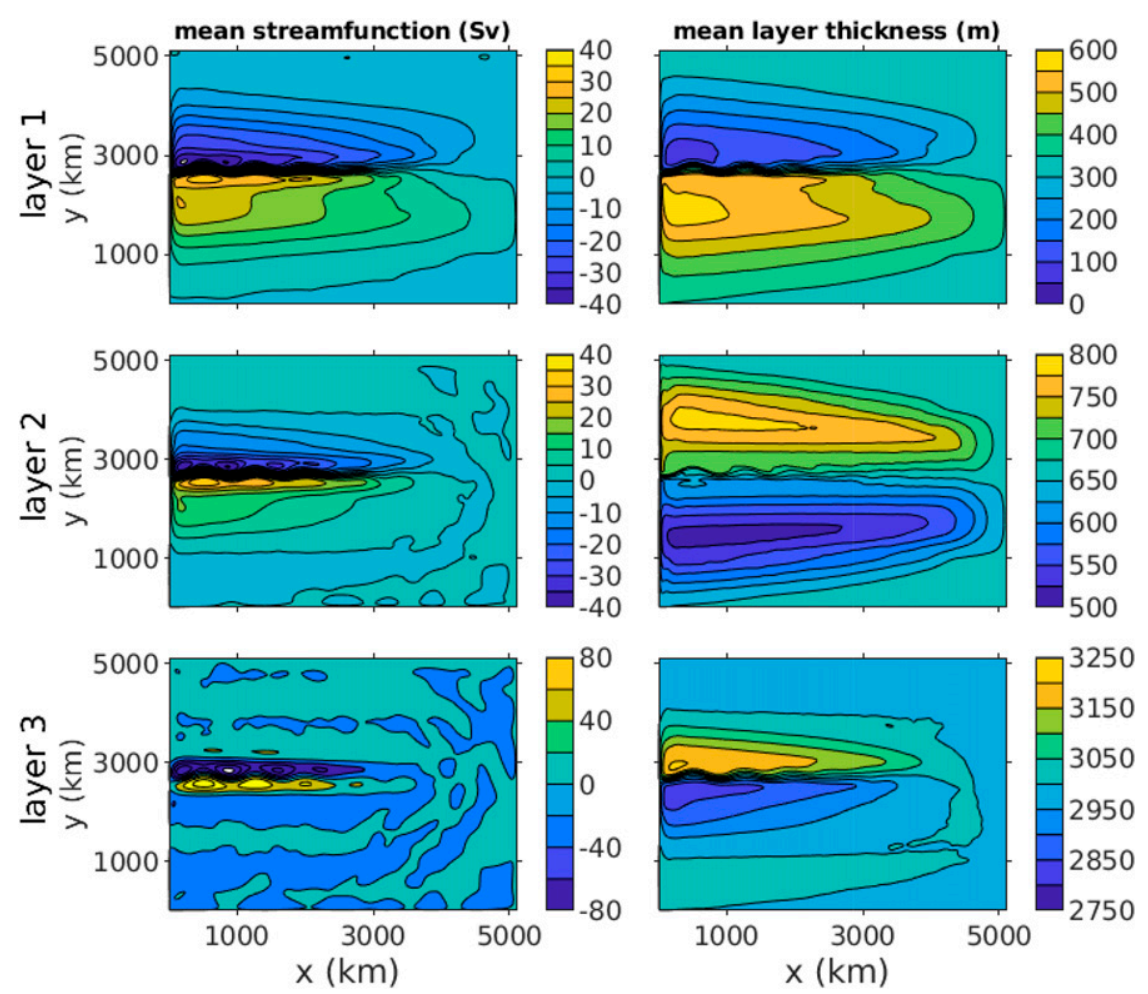

FIG. 1. Mean (left) streamfunction (Sv) and (right) layer thickness (m) for the quasigeostrophic model run for 120 years.

wind-driven Sverdrup flow is carried in the upper layer with maximum gyre transports of $30 \mathrm{~Sv}\left(1 \mathrm{~Sv} \equiv 10^{6} \mathrm{~m}^{3} \mathrm{~s}^{-1}\right)$. There are also very narrow recirculations adjacent to the separated jet. The middle layer also has gyre-scale recirculations forced by baroclinic instability of the separated jet and gyres (there is no direct wind forcing in this layer), as well as the narrow recirculations. The deep layer is dominated by the narrow recirculation gyres, demonstrating their barotropic structure. Detailed analysis of the dynamics of similar wind-driven gyres can be found in Berloff (2016) and Berloff (2018).

The mean thickness of the upper layer varies from about $600 \mathrm{~m}$ in the subtropical gyre to $100 \mathrm{~m}$ in the subpolar gyre, reflecting the large-scale Sverdrup flow (Fig. 1). The narrow barotropic recirculation gyres are not evident in the layer thickness. The middepth layer thickness mirrors that of the upper layer, thin in the subtropical gyre and thick in the subpolar gyre. The bottom layer shows a similar structure with a thinner subtropical gyre and a thicker subpolar gyre, indicating that there is also some baroclinic shear between layers 2 and 3.

To use the QG model to study the MOC, first step is to calculate the MOC from the quasigeostrophic variables. The streamfunction $\psi$ represents only the geostrophic velocity field, and so does not include ageostrophic effects due to time dependence, variations in the Coriolis parameter, or nonlinearities in the momentum balance. Furthermore, the streamfunction is constant on the sidewalls, as required to impose the no-normal flow condition on the geostrophic velocity.
However, the geostrophic component of the MOC is driven by the pressure difference between the eastern and western boundaries. The primitive equations allow ageostrophic terms that balance the pressure gradient on the boundary, thus supporting a change in pressure across the domain and an interior geostrophic meridional transport while satisfying the nonormal-flow boundary condition.

The MOC can be inferred from the evolution of the layer thicknesses at each latitude. The layer thicknesses, derived from the thermal wind relation between each layer, are written as

$$
\begin{aligned}
& h_{1}=H_{1}+\left(f_{0} / g_{12}\right)\left(\psi_{1}-\psi_{2}\right), \\
& h_{2}=H_{2}+\left(f_{0} / g_{12}\right)\left(\psi_{2}-\psi_{1}\right)+\left(f_{0} / g_{23}\right)\left(\psi_{2}-\psi_{3}\right), \quad \text { and } \\
& h_{3}=H_{3}+\left(f_{0} / g_{23}\right)\left(\psi_{3}-\psi_{2}\right) .
\end{aligned}
$$

To close the mass budget in each layer, the time rate of change of the zonal integral of the layer thickness must be provided by a divergence of the meridional mass flux. The MOC in each layer, $\Psi_{k}$, can thus be defined as the zonal and meridional integral of the time rate of change of layer thickness:

$$
\Psi_{k}(y)=\int_{0}^{y} \int_{0}^{L} h_{k t} d x d y .
$$

The boundary condition of no flow through the southern boundary is imposed, $\Psi_{k}(0)=0$. Note that, because the model is adiabatic and in a closed basin there is no mean MOC. For 

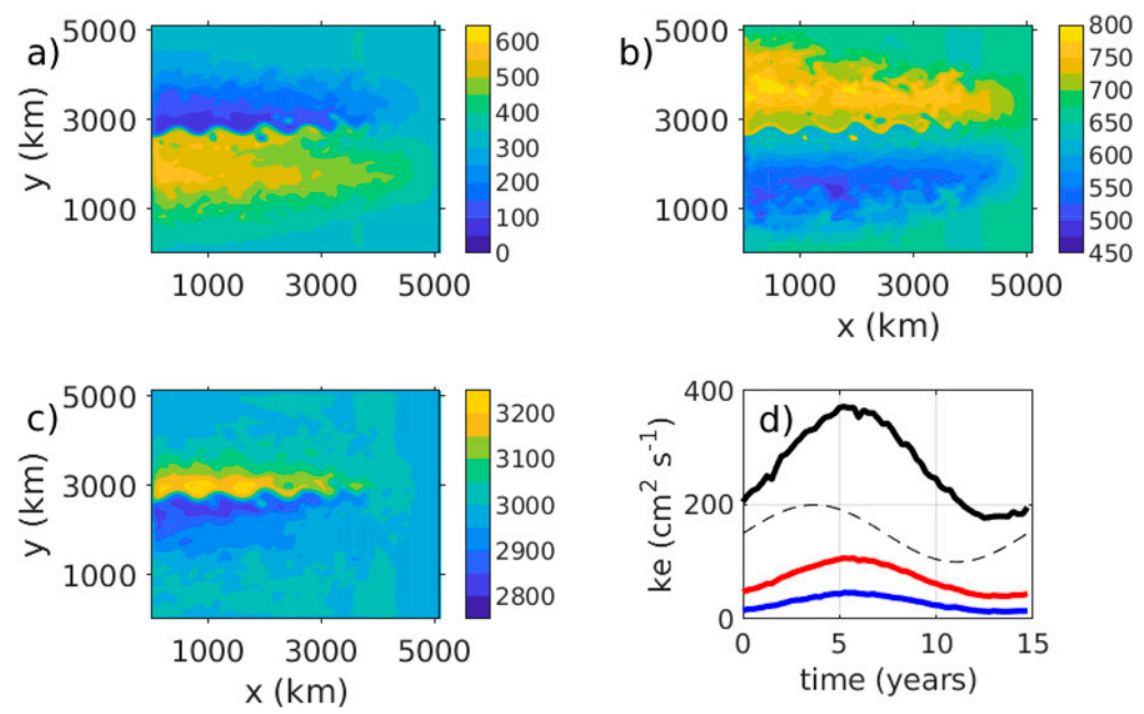

FIG. 2. Snapshot of layer thickness (m) for year 74 for (a) layer 1, (b) layer 2, and (c) layer 3. (d) Kinetic energy in each layer, averaged into the 15 -yr forcing period. black, layer 1; red, layer 2; blue, layer 3. the dashed line is the phase of wind stress (arbitrary units).

time-dependent winds the subtropical and subpolar gyres will adjust through the upper layer spinning up and down as the winds vary. However, the consequences of the upper layer fluctuations are felt throughout the water column, as required to conserve mass in each layer. For example, if the wind strengthens, the subtropical gyre will deepen and the subpolar gyre will shoal. Because the system is adiabatic, the some of the layer-2 water displaced from the deepening subtropical gyre must flow across the gyre boundary to fill the thickening layer 2 in the shoaling subpolar gyre. This adjustment process is modulated by baroclinic waves with westward phase and group velocities, making the response a function of the stratification and forcing frequency.

An example of the wind-driven variability of the MOC is provided by a calculation with $\tau_{0}=0.12 \mathrm{~N} \mathrm{~m}^{-2}, \tau^{\prime}=0.4, y^{\prime}=$ 0 , and a forcing period of $15 \mathrm{yr}\left(\omega=1.33 \times 10^{-8} \mathrm{~s}^{-1}\right)$. A snapshot of the layer thicknesses on year 74 is shown in Fig. 2. The separated jet is meandering and shedding warm and cold core eddies. The meanders and eddies penetrate through all three layers, although they are surface intensified. The westward flow along the northern subpolar and southern subtropical gyres is also dominated by meanders and eddies. This is evidence of baroclinic instability of the gyre circulation and will be shown below to have an important influence on the MOC. The basin-averaged kinetic energy, averaged into the periodic forcing period of $15 \mathrm{yr}$, is shown in Fig. 2d. The signal is largest in the upper layer but, as a fraction of the mean kinetic energy, it is largest in the deep layer. The periodic cycle in kinetic energy lags the that for the wind stress by several years.

The MOC diagnosed at the midlatitude of the basin in each layer from (11) is shown in Fig. 3. The meridional transport in layer 1 (black) is very close to the Ekman transport (green) with amplitude of $2.45 \mathrm{~Sv}$. Because the domain is closed and the sea surface is very stiff, the transport in the middle and deep layers must compensate for the upper layer transport. The transport in the deep layer is nearly of equal magnitude and $180^{\circ}$ out of phase with the upper layer transport. The transport in the middle layer is weaker and approximately $90^{\circ}$ out of phase with the upper layer transport. The smaller magnitude is not surprising given that layer 2 is much thinner than layer 3; however, the phase shift suggests a more complex response than the simple barotropic return flow discussed by Bryan (1982) and Jayne and Marotzke (2001).

\section{A simple quasigeostrophic theory}

Insight into the basic response of the midlatitude stratified ocean to time-dependent winds is provided by a simple analytic solution to the quasigeostrophic equations subject to periodic forcing. The starting point is the linear quasigeostrophic potential vorticity equation:

$$
\frac{f_{0}^{2}}{N^{2}} \psi_{z z t}+\beta \psi_{x}=0
$$

The long-wave approximation is made so that relative vorticity is neglected. The mean ocean state is also at rest, so the mean wind-driven subtropical and subpolar gyres are not included. Solutions for the streamfunction are sought that are wave-like in the zonal direction with wavelength $2 \pi / \lambda$ and frequency $\omega$ with a vertical structure $\phi$. It is assumed that the stratification is uniform so that the vertical structure function $\phi$, derived from (12), is a simple cosine form:

$$
\psi_{n}=e^{i\left(\lambda_{n} x-\omega t\right)} \phi_{n}(z), \quad \phi_{n}=2^{1 / 2} \cos (n \pi z / H) .
$$

The wavenumber is related to the frequency through the dispersion relation 


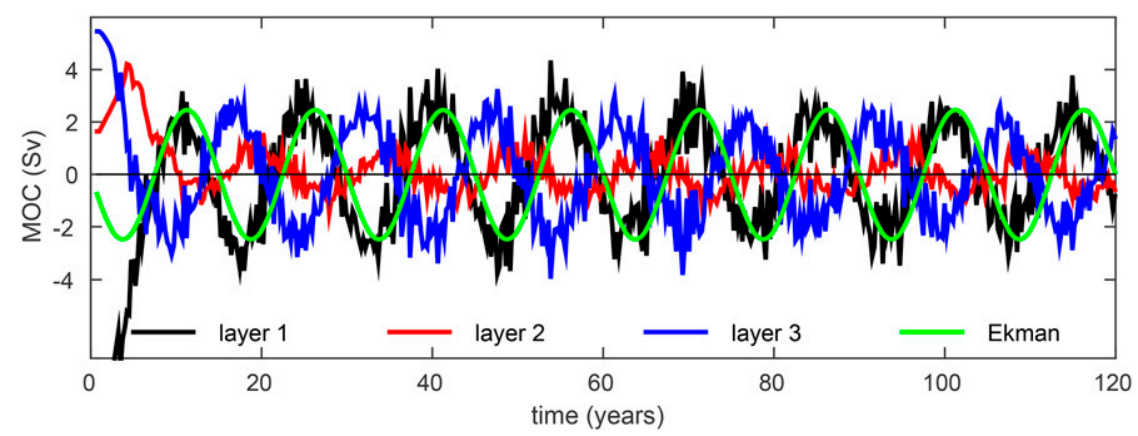

FIG. 3. Time series of the meridional overturning circulation in each layer and the meridional Ekman transport anomaly.

$$
\lambda_{n}=-\omega /\left(\beta L_{n}^{2}\right), \quad L_{n}=N H /\left(n \pi f_{0}\right),
$$

where $L_{n}$ is the baroclinic deformation radius for mode $n$. The bottom depth is $H$, and the scale factor of $2^{1 / 2}$ is chosen to simplify the solution for $\psi$. There are an infinite number of vertical modes indicated by mode number $n$. The complete streamfunction is then the sum over all modes:

$$
\psi=\sum_{n} \phi_{n}(z) \psi_{n}(x, y, t)
$$

Multiplying (12) by $\phi_{n}$ and integrating in $z$ from $-H$ to 0 yields an equation for $\psi_{n}$. Using (13) and (7a) and integrating by parts twice gives

$$
\psi_{n t}-\beta L_{n}^{2} \psi_{n x}=\frac{f_{0} L_{n}^{2} \phi_{n}(0)}{H} W_{E}(y) \sin (\omega t)
$$

The Ekman pumping boundary condition at the surface enters through the limits of one of the integration by parts. The value of the vertical mode at the surface is $\phi_{n}(0)$ and $W_{E}(y)$ is the pattern of the Ekman pumping velocity at the surface.

For the case in which the wind pattern is constant $\left(y^{\prime}=0\right)$ but the magnitude varies $\left(\tau^{\prime}>0\right)$, the Ekman pumping is simply

$$
W_{E}=\frac{\pi \tau_{0} \tau^{\prime}}{\rho_{0} f_{0} L_{g}} \sin \left[\pi\left(y-y_{0}\right) / L_{g}\right] .
$$

The case with a shifting wind pattern is more complex, but a useful approximation is obtained for the local rate of change of the Ekman pumping as $\tau_{t} \approx \tau_{y} y_{t}$, which gives

$$
W_{E}=-\frac{y^{\prime} \pi^{2} \tau_{0}}{\rho_{0} f_{0} L_{y}^{2}} \cos \left[\pi\left(y-y_{0}\right) / L_{g}\right]
$$

The fundamental change relative to the case with changing wind strength is that the Ekman pumping anomaly is a maximum at the gyre boundary while for the changing wind strength it is a maximum at the centers of the gyres.

The solution to (16), subject to $\psi=0$ at the eastern boundary, is

$$
\psi_{n}=-A \frac{\pi L_{n}^{2} \tau_{0} \phi(0)}{\rho_{0} L_{g} H \omega}\left[\sin \left(\lambda_{n} x-\omega t\right)+\sin (\omega t)\right] \phi_{n}(z),
$$

which may also be written as

$$
\psi_{n}=-A \frac{2 \pi L_{n}^{2} \tau_{0} \phi(0)}{\rho_{0} L_{g} H \omega} \sin \left(\lambda_{n} x / 2\right) \sin \left(\lambda_{n} x / 2-\omega t\right) \phi_{n}(z)
$$

where the value of $A$ depends on which forcing function is used. This solution is similar in form to the single-layer solution of White (1977) but here the depth-dependent total response is the sum over all modes. For strengthening/weakening winds,

$$
A=\tau^{\prime} \sin \left[\frac{\pi\left(y-y_{0}\right)}{L_{g}}\right]
$$

and, for a shifting wind pattern,

$$
A=\frac{\pi y^{\prime} L}{L_{g}} \cos \left[\frac{\pi\left(y-y_{0}\right)}{L_{g}}\right] .
$$

The time rate of change of the isopycnal surfaces is given by $\left(f_{0} / N^{2}\right) \psi_{z t}$, which, making use of (13), is for each mode

$h_{n t}=-A \frac{4 \tau_{0}}{n \rho_{0} L_{g} f_{0}} \sin \left(\lambda_{n} x / 2\right) \cos \left(\lambda_{n} x / 2-\omega t\right) \sin (n \pi z / H)$.

The MOC is then derived by integrating $h_{n t}$ in the zonal and meridional directions for each vertical mode in the same manner as for the diagnostic in the QG model (11). The total MOC is simply the sum over all modes considered. The zonal integral is taken from the eastern boundary to the western boundary, which lies at $x=-L=-5120 \mathrm{~km}$. The expression for $h_{n t}$ indicates that the MOC is not explicitly dependent of the forcing frequency $\omega$, although the wavenumber $\lambda_{n}$ does depend on $\omega$ through the dispersion relation (14).

The amplitude decreases with increasing mode number and with decreasing Ekman pumping. Its horizontal structure is that of a standing wave with wavelength $4 \pi / \lambda_{n}$ with a wave that travels to the west with phase speed 2 times that for a long Rossby wave. Note that the interface displacement is always zero on the eastern boundary $(x=0)$, while that on the western boundary $(x=-L)$ will fluctuate in time, depending on the forcing frequency and the basin width. This is consistent with the findings of the RAPID-MOCHA array that variations in 
a)

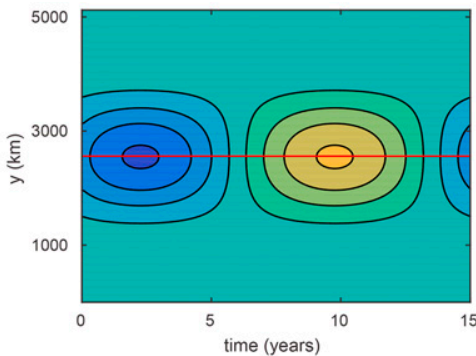

c)

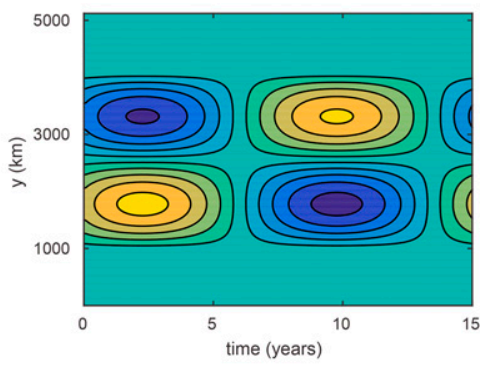

b)

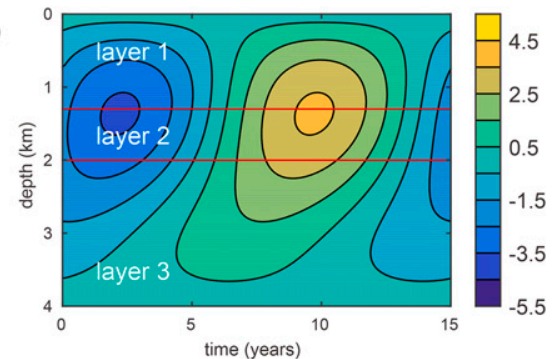

d)

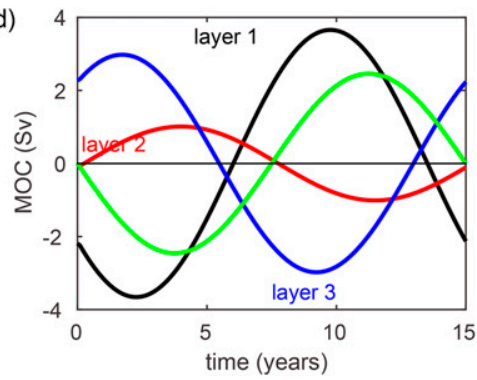

FIG. 4. MOC from the analytic quasigeostrophic theory. (a) MOC (Sv) as a function of time and latitude in the upper layer [at the depth of the upper red line in (b)] for the case of oscillating wind strength with 15 -yr period; (b) vertical structure of the MOC (Sv) at the gyre boundary as a function of time [red line in (a)]; (c) MOC (Sv) as a function of latitude and time for the case of oscillating wind stress latitude; (d) MOC at the gyre boundary in each layer [as shown in (b)] as a function of time for the case with oscillating wind strength. The green line is the Ekman transport.

the MOC are dominated by variations in the layer thickness on the western boundary (Frajka-Williams et al. 2016).

The meridional structure of the MOC as a function of time for varying wind strength is shown in Fig. 4 a for the case with a 15-yr periodic forcing, $\tau_{0}=0.12 \mathrm{~N} \mathrm{~m}^{-2}, \tau^{\prime}=0.4$, and two vertical modes. This is taken at the depth of the maximum MOC, indicated by the upper red line in Fig. 4b. As the wind strengthens the upper layer MOC strengthens to the south, reflecting the increasing southward transport in the Ekman layer. The meridional distribution shows that this is a flux of mass from the subpolar to the subtropical gyre. There is no influence outside the latitude range of the wind stress curl anomalies.

The vertical structure of the MOC at the gyre boundary (red line in Fig. 4a) is shown in Fig. 4b. The MOC is surface intensified with a phase shift in the vertical. For purposes of comparison between the continuously stratified theory and the three-layer QG model, three layers have been identified in the theory, indicated by the red lines in Fig. 4b. The MOC within these three layers is plotted in Fig. 4d. The magnitude and phase predicted by the theory compares well to that found in the numerical model (Fig. 3). The deep layer slightly leads and is a little stronger than the Ekman transport. The upper layer and deep layer are of similar magnitude and nearly $180^{\circ}$ out of phase while the middle layer is weaker and lags the lower layer by about $90^{\circ}$. The agreement is encouraging given that the model has a simple three-layer vertical structure, mean subtropical and subpolar gyres, imposes no-normal flow boundary conditions on all boundaries, and includes relative vorticity.
The horizontal pattern of the MOC in the upper layer for the case with shifting winds is shown in Fig. 4c. In this case the MOC does not cross the gyre boundary and the mass redistribution is confined within each gyre. The magnitude and phase are very similar to the case for varying wind strength. The vertical structure is the same as is shown in Fig. $4 \mathrm{~b}$.

\section{Parameter dependence and role of nonlinearities}

\section{a. Eddy effects}

The basic structure of the wind-driven MOC predicted by the theory is now compared with that produced by linear and nonlinear QG model simulations with 15 -yr forcing period. The linear model runs are identical to the nonlinear runs except the advection of relative vorticity is set to zero. The results for both higher- and lower-frequency forcing will be discussed in the next subsection. The linear model result shows a very similar latitude/time dependence as predicted by the theory (Fig. 5, left column). The primary balance is between the upper and deep layers with the middle layer of lower amplitude and lagging the deep layer by $90^{\circ}$, consistent with the theory in section 4. There is no MOC outside the latitude range of the wind anomalies. This is different from what is found for the primitive equations, for which the Rossby wave speed varies as a function of latitude. Spall and Nieves (2020) demonstrated that an imbalance between the adjustment in the subtropical and subpolar gyres due to the meridional dependence in the baroclinic Rossby wave speed forces an MOC anomaly that 

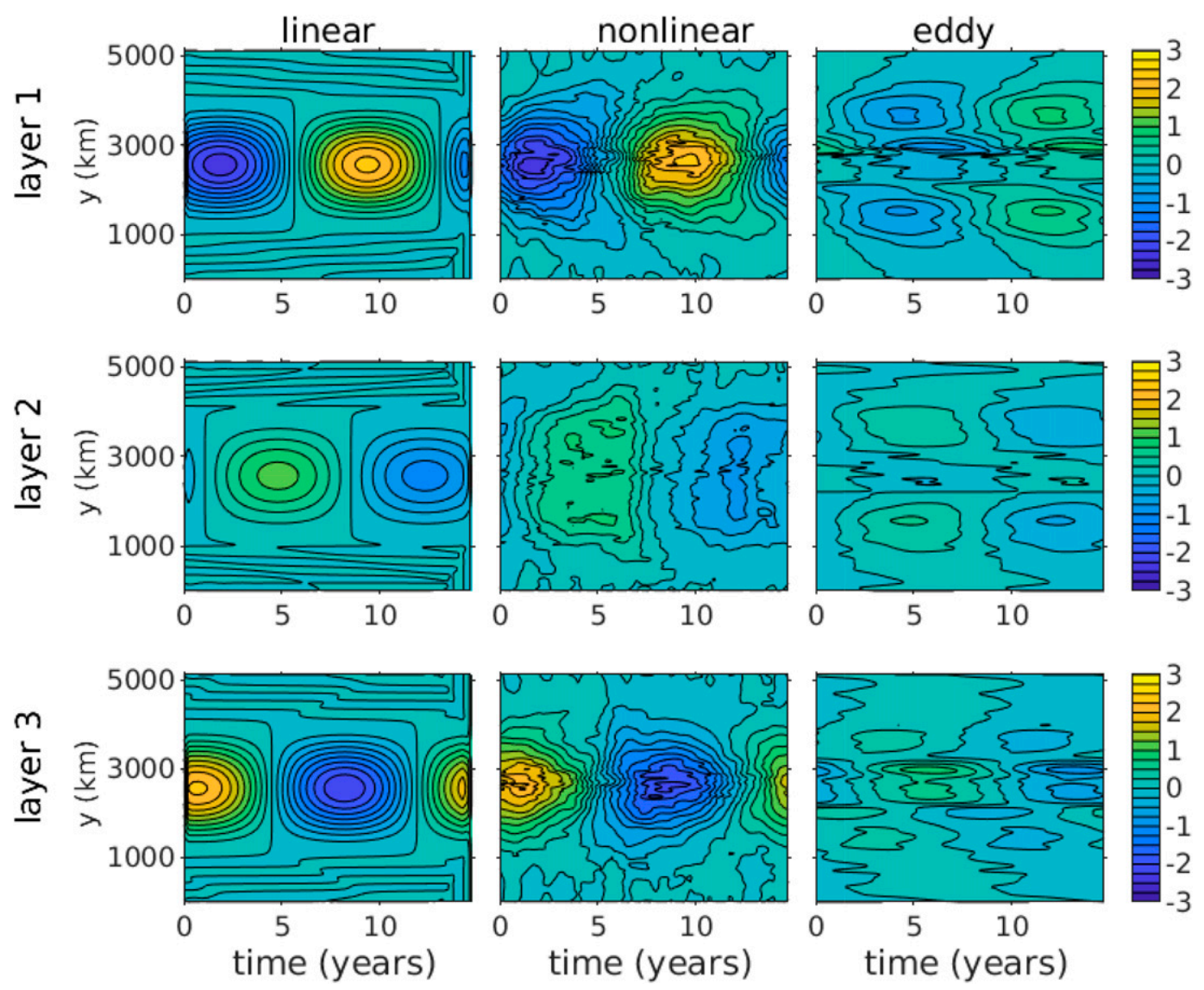

FIG. 5. MOC as a function of time and latitude for the (left) linear model and (center) nonlinear model and (right) due to geostrophic eddies for each model layer for the case of oscillating wind strength with 15-yr period.

extends well south of the subtropical gyre, even into the Southern Hemisphere. We see no such remotely forced MOC here because in QG physics the Rossby wave speed is independent of latitude.

The nonlinear model result has a broadly similar pattern, but the MOC extends to higher and lower latitudes and, in the middle layer, is weaker near the gyre boundary and stronger both north and south of the direct wind forcing (Fig. 5, middle column). This difference is largely due to eddy fluxes.

The meridional overturning driven by geostrophic eddies can be calculated directly from the layer thickness and streamfunction as

$$
\Psi_{k}^{\prime}(y)=\int_{0}^{y} \int_{0}^{L} \overline{\psi_{k x}^{\prime} h_{k y}}-\overline{\psi_{k y}^{\prime} h_{k x}} d x d y
$$

where the overbar indicates the time average and primes are deviations from the time mean. This does not include all eddy effects, just those associated with geostrophic eddies. The eddy fluxes are largest on the northern side of the subpolar gyre and the southern side of the subtropical gyre (Fig. 5, right column). This is where the westward flanks of the gyres are unstable and the eddy thickness fluxes are important all along the zonal extent of the gyres (Fig. 2). The sense of the eddy-induced
MOC is to flatten the isopycnals at the northern and southern limits of the wind forcing. The primary eddy-driven cell at this forcing frequency is between the upper and middle layers with a weaker signal in the deepest layer that is of the same sign as that in the middle layer. This is expected from baroclinic instability because the baroclinic shear is largest between layers 1 and 2 but there is still some shear between layers 2 and 3. The eddy fluxes lag the upper layer MOC and wind stress curl by several years. This is because, as the wind stress curl changes say from strong to weak, the gyres are stronger than can be balanced by the wind. As a result, eddies are able to relax the isopycnal slopes during these periods of forcing decay. A similar, but opposite, effect occurs while the winds are strengthening. The largest impact is on the middle layer, where the MOC near the gyre boundary is weakened and the MOC is extended poleward and equatorward of the wind forcing.

The basic pattern predicted by the theory (Fig. 4c) for the case of shifting winds is also reproduced in the numerical model (Fig. 6). The clean boundary between the subpolar and subtropical gyres predicted by the theory is not found in the model because the wind stress curl at the gyre boundary in the theory is always zero due to the first-order expansion of $\tau_{t}$ while in the model it takes both positive and negative values as the 

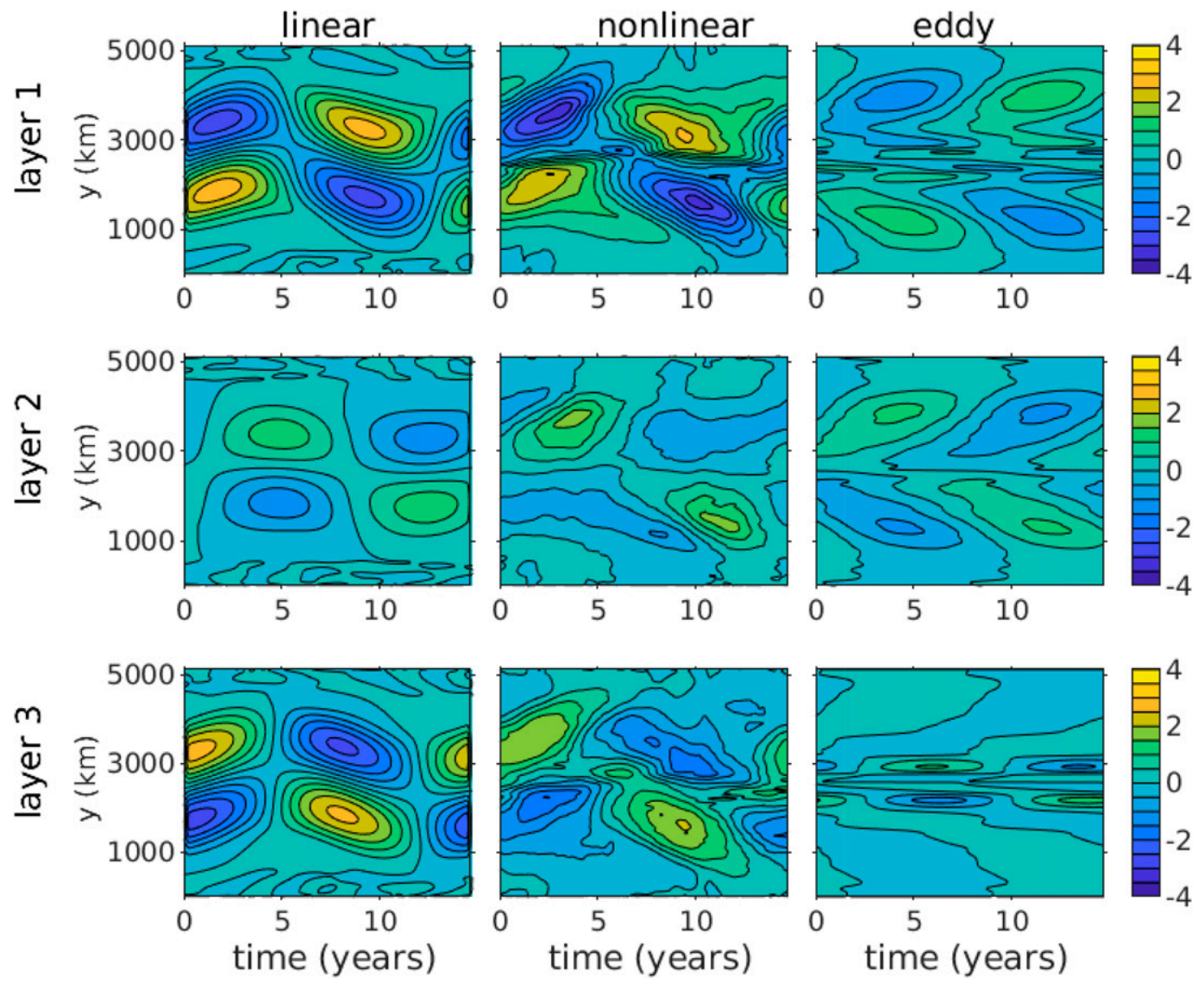

FIG. 6. As in Fig. 5, but for the case of oscillating wind latitude with $y^{\prime}=0.1$.

wind oscillates. However, the general pattern and amplitude of MOC cells isolated within each gyre are reproduced in the model. The nonlinear result is again similar with cells extending beyond the latitude range of the wind stress. Eddies are of similar importance and primarily located on the poleward and equatorward flanks of the gyres. The eddy-driven cells show a phase shift with latitude, which reflects the shifting location of the increase/decrease in wind stress curl.

\section{b. Frequency dependence}

The dependence of the MOC on forcing frequency is now evaluated by analyzing a series of model runs with forcing periods of $1,2,5,10,15,20$, and 40 years. In each case the model is run for 20 years before the diagnostics are calculated. In the nonlinear runs the model duration is for 6 forcing periods beyond this 20 -yr spinup period (except the 40 -yr period, which was run for 3 forcing periods). This allows for some temporal averaging of mesoscale eddy and interannual variability to isolate the periodic response. In the diagnostics below, the MOC is calculated using (11), then averaged over the duration of the post spinup model run into an average cycle at the forcing frequency, and finally the amplitude and phase are determined by a spectral fit at the forcing frequency at each latitude. An additional set of calculations with time varying wind strength were carried out with $\beta=1 \times 10^{-11} \mathrm{~m}^{-1} \mathrm{~s}^{-1}$, so the basin-crossing time scale was 2 times that for the runs diagnosed here. The results were generally consistent in terms of the behavior in the forcing period scaled by the basin-crossing time scale, and so are not reported separately here.

The amplitude of the MOC as a function of latitude and forcing period is shown in Fig. 7 for the theory (left column), linear model (middle column), and nonlinear model (right column) for each of the layers. In each case, the amplitude has been scaled by the maximum amplitude in layer 1 , which are $4.7,2.8$, and $2.9 \mathrm{~Sv}$ for the theory, linear model, and nonlinear model. Thus, the theory overpredicts the amplitude of the MOC by about $50 \%$, as evident in Figs. 3 and 4. The forcing period has been scaled by the time it takes for the first-mode baroclinic Rossby wave to cross the basin $\left[T=L /\left(\beta L_{1}^{2}\right)=5.1 \mathrm{yr}\right]$. The theory predicts a primary balance between the upper and deep layers, as expected from the larger layer thickness of the deep layer relative to the middle layer. At short forcing periods $[2 \pi /(\omega T)<1]$ the ratio of the MOC in layers 2 and 3 is close to the ratio of their thicknesses. This results from a nearly in-phase, barotropic return flow below layer 1 . The amplitude shows a weak local maximum near $2 \pi /(\omega T)=2$ and a weak local minimum near $2 \pi /(\omega T)=1$. At lower-frequency forcing, $2 \pi /(\omega T)>2$, the amplitude begins to decrease in layer 3 and increase in layer 

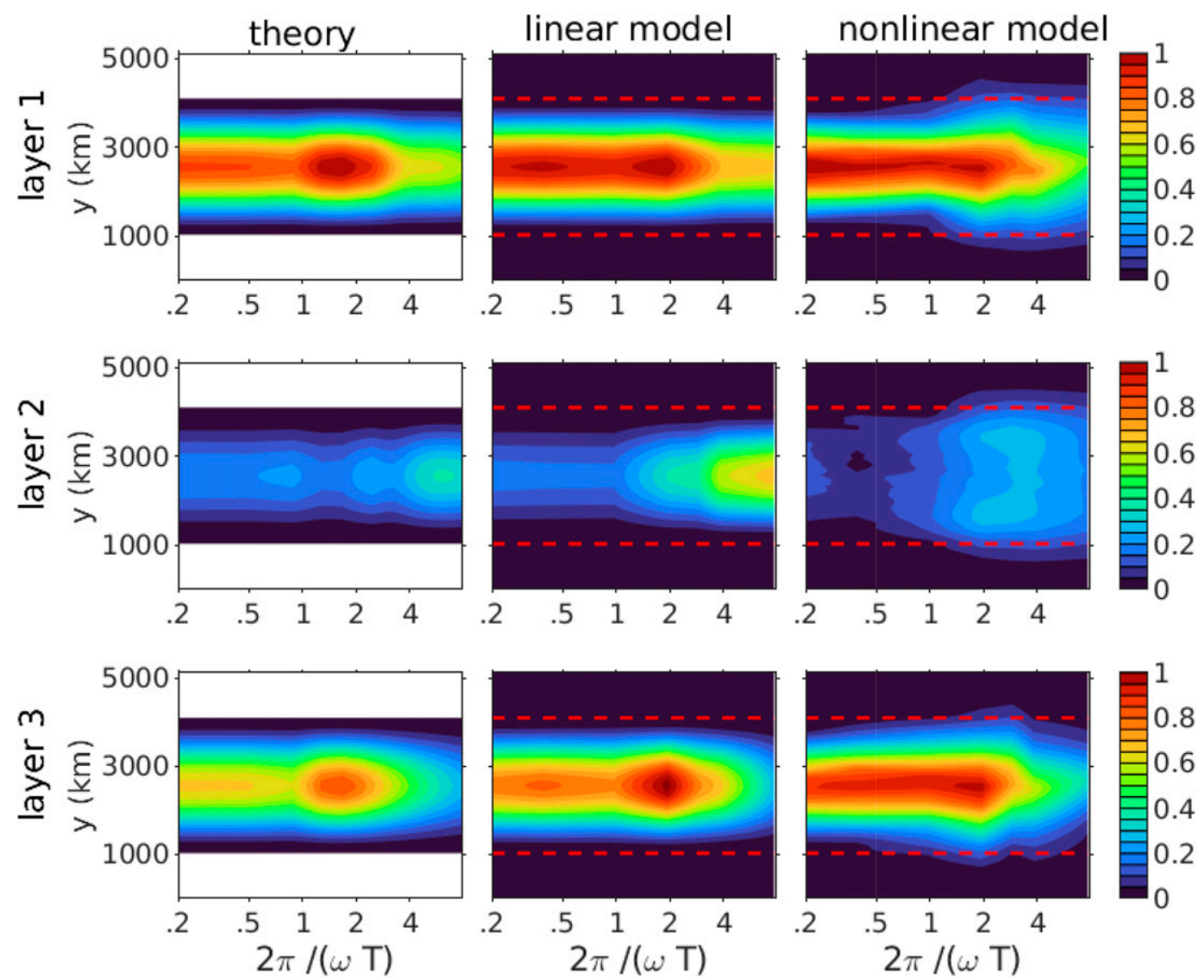

FIG. 7. Magnitude of the MOC variability as a function of latitude and forcing period (scaled by the first-mode baroclinic Rossby wave basin crossing time scale) for (left) theory and the (center) linear model, and (right) nonlinear model for each layer and oscillating wind strength. For ease of comparison, in each case the amplitude has been scaled by the maximum amplitude in layer 1.

2. At such low frequencies the first baroclinic mode has crossed the basin and left behind a nearly equilibrated circulation (Anderson and Gill 1975). However, the second baroclinic mode is still crossing the basin.
The zero crossing of the first baroclinic mode lies at the interface between layers 2 and 3 (Fig. 8a). In fact, this is a physically based means to identify the interface between the deep and middle layers. As a result, the first mode projects a)

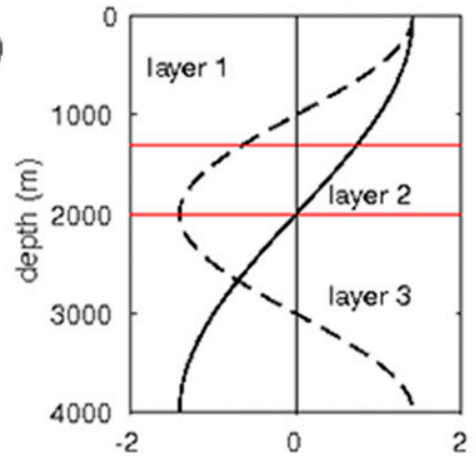

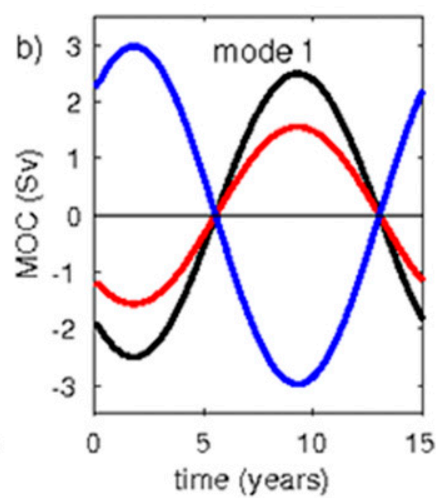

FIG. 8. (a) First (solid) and second (dashed) baroclinic modes used in the theory along with the interfaces used to define three layers for comparison with the numerical model. Also shown is MOC at the gyre boundary forced by (b) the first baroclinic mode and (c) the second baroclinic mode. The black line is the upper layer, the red line is the middle layer, and the blue line is the deep layer. 

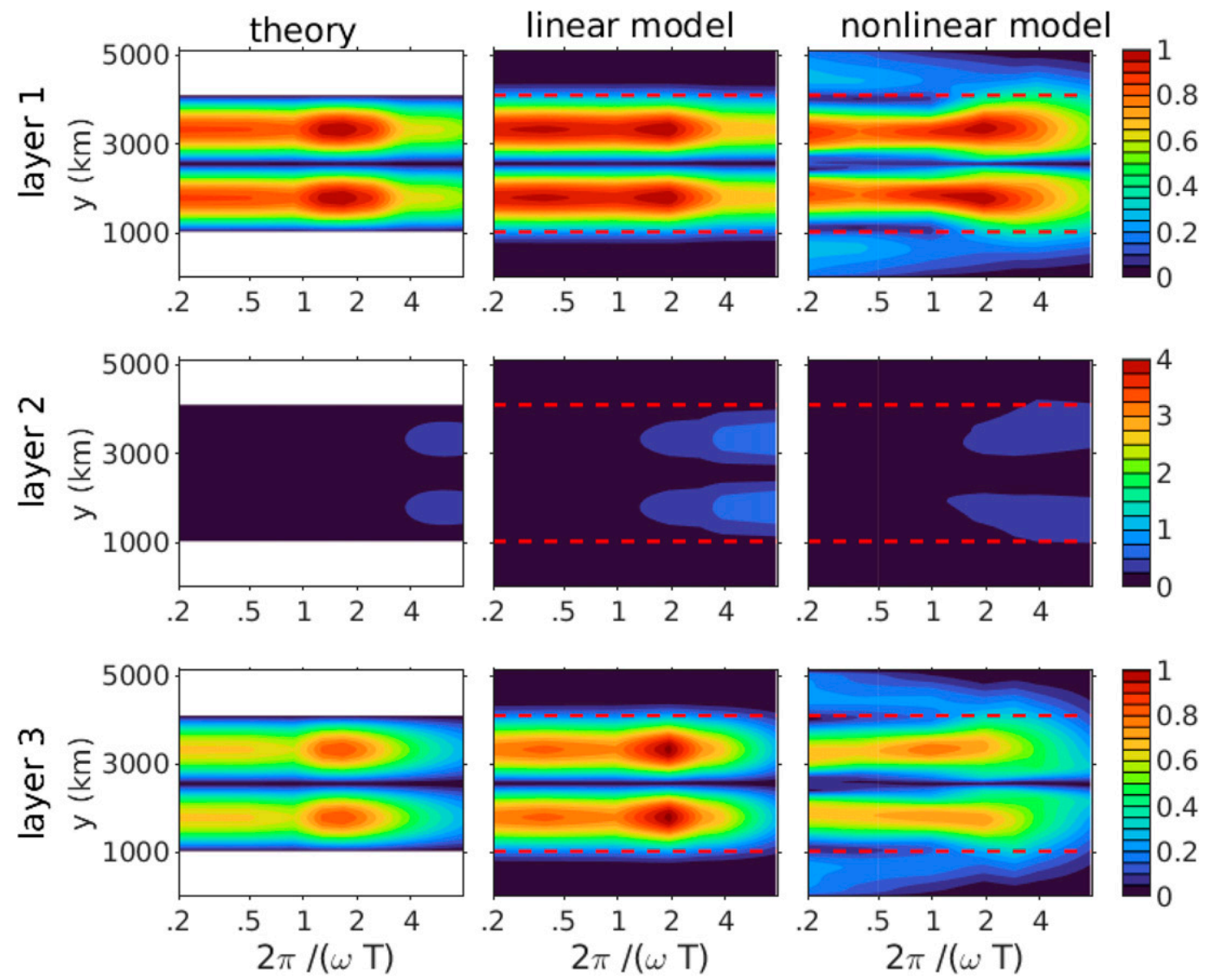

FIG. 9. As in Fig. 7, but for the case of oscillating wind latitude.

cleanly onto layers 1 and 2 moving in one direction and layer 3 moving in the opposite direction (Fig. 8b). However, the second baroclinic mode integrates to zero in the deep layer while the upper and middle layers are nearly equally opposed. The MOC forced by propagation of the second baroclinic mode is zero in the deep layer and in the opposite direction to the first baroclinic mode in the middle layer. This is the fundamental reason that the middepth MOC becomes out of phase with the upper and lower layers at forcing periods longer than $T$. The amplitude of the MOC can exceed that of the Ekman pumping because the rate of change of the volume in layer 1 can be forced not only from above by Ekman pumping but also from below by changes in the thickness of layer 2 . Consideration of the second baroclinic mode allows for layer 2 to become phase-shifted from the Ekman pumping, thus enhancing the MOC in certain frequency bands. At high-frequency forcing there is no phase shift in the vertical and the transport in layer 2 is dominated by the second baroclinic mode, and hence in the same direction as that in layer 3 and opposite to that in layer 1.

At forcing periods much longer than $T$, the wavelength of the first-mode baroclinic Rossby wave exceeds the width of the basin, $\lambda_{1} L \rightarrow 0$. The MOC forced by the first mode, which is proportional to the zonal integral of $\sin \left(\lambda_{1} x / 2\right)$ from (23), decreases. However, the second mode is slower and thus still contributes to the interface displacement and the MOC. So at low frequencies the MOC becomes more surface intensified and confined between layers 1 and 2 .

The amplitude of the MOC for the linear QG model as a function of latitude and forcing period is shown in Fig. 7 in the middle column. The model shows a very similar pattern to the theory, although the amplitude in the upper and deep layers is approximately $50 \%$ lower. However, given the continuous, uniform stratification in the theory and the three-layer representation in the model it is not surprising that there are some differences in the magnitude. The red dashed lines mark the meridional limits of the wind forcing, demonstrating that the variability does not extend to latitudes outside the region of forcing. We see a similar local maximum around $2 \pi /(\omega T)=2$ and a shift of the MOC from a deep structure to a shallow structure as the forcing period is increased beyond the basincrossing time scale.

The amplitude of the MOC for the nonlinear QG model as a function of latitude and forcing period is shown in Fig. 7 in the right hand column. The overall structure is similar, although the amplitudes and meridional extent differ somewhat from the linear result. At high-frequency forcing the deep MOC is stronger than that in the linear model and the middepth MOC is weaker. This is a result of eddy fluxes relaxing the interface slope between layers 2 and 3. The largest difference is in the 

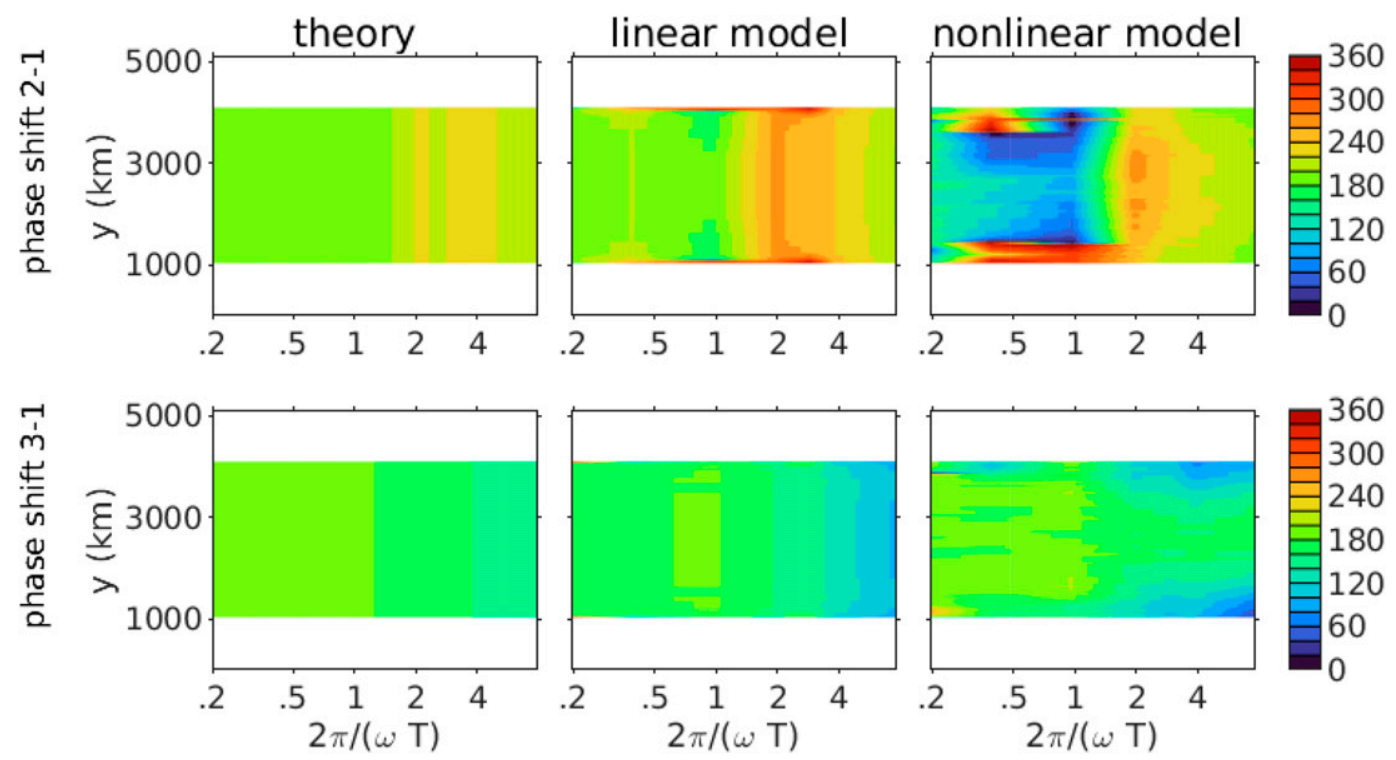

FIG. 10. Phase of layers (top) 2 and (bottom) 3 relative to the upper layer for the (left) theory, (center) linear model, and (right) nonlinear model.

layer $2 \mathrm{MOC}$ at low-frequency forcing, where the nonlinear model shows a much weaker response. The variability in the MOC at low frequencies also extends to higher and lower latitudes than in the linear model or the theory. So nonlinearities do not change the fundamental response to changing wind strength but they do alter the amplitude and meridional extent, particularly at middepths.

The same diagnosis for the shifting wind cases is shown in Fig. 9. As expected from the theory, the MOC for these cases does not cross from the subpolar to the subtropical gyre. Instead, we see a structure similar to that for the wind strengthening/weakening case confined within each gyre. There is again qualitative agreement between the theory and linear model. Nonlinearities weaken the middepth MOC at high frequencies and spread the MOC to higher and lower latitudes at low frequencies in all layers.

\section{c. Phase and correlation}

The coupling between layers is further revealed by the dependence of phase and correlation between layers on latitude and forcing period. Figure 10 shows that the theory predicts the middle and deep layers to be $180^{\circ}$ out of phase with layer 1 at high-frequency forcing. There is a phase shift in the middle layer for $2<2 \pi /(\omega T)<4$. This is the period for which the first baroclinic mode has crossed the basin but the second baroclinic mode has not. This is the phase shift evident in Fig. 4 for the 15 -yr forcing period. At longer periods layer 2 is again $180^{\circ}$ out of phase with layer 1 . Layer 3 is still dominated by the mode 1 wave (albeit at smaller amplitude due to the low-frequency forcing), so it is now phase shifted from layer 1 , which is more strongly coupled to layer 2 . The linear model shows similar patterns to that predicted by the theory with slightly larger phase shifts. However, the nonlinear model shows a much different pattern. There is a phase shift in layer 2 for $2<2 \pi /(\omega T)<4$, but at intermediate and higher frequencies the phase lag between layers 2 and 1 is much less than in the linear models. At these frequencies baroclinic instability derives from the shear between layers 2 and 3. Layer 3 is out of phase with layer 1, and so the eddy fluxes drive layer 2 to be more in phase with layer 1 . The deep layer remains $180^{\circ}$ out of phase with layer 1 near the gyre boundary at all frequencies due to the deep eddy-driven MOC (lower-right panel in Fig. 10).

The correlation between each of the layers is shown in Fig. 11 as a function of latitude and forcing period. The linear theory predicts that layers 2 and 3 are strongly correlated with each other and anticorrelated with layer 1 at high-frequency forcing, consistent with the $180^{\circ}$ phase lag. The phase shift in layer 2 for $2<2 \pi /(\omega T)<4$ reduces the correlation with layer 1 , as expected. Layer 3 shows a very slight phase shift at very lowfrequency forcing relative to layer 1 . Similar patterns are found for the linear model, although the model produces a larger loss of correlation at low frequencies due to the larger phase shift seen in Fig. 10. The correlation is independent of latitude in the theory and nearly so in the linear model. The nonlinear model produces a loss of correlation between layers 1 and 2 at all frequencies and at all latitudes with a maximum loss at intermediate frequencies and in the midlatitudes of the gyres. The eddy coupling maintains the anticorrelation between layers 1 and 3 at low-frequency forcing. This is consistent with the observed weak correlation between the Ekman transport and Upper North Atlantic Deep Water and the strong correlation between the Ekman transport and Lower North Atlantic Deep Water (Frajka-Williams et al. 2016).

\section{d. Meridional coherence}

The meridional coherence of the wind-driven variability of the MOC is indicated in Fig. 12 as a function of latitude and 

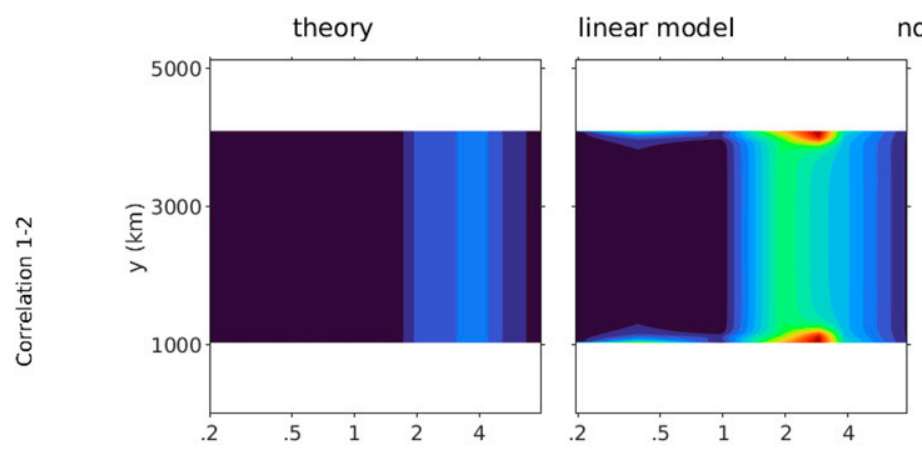

nonlinear model
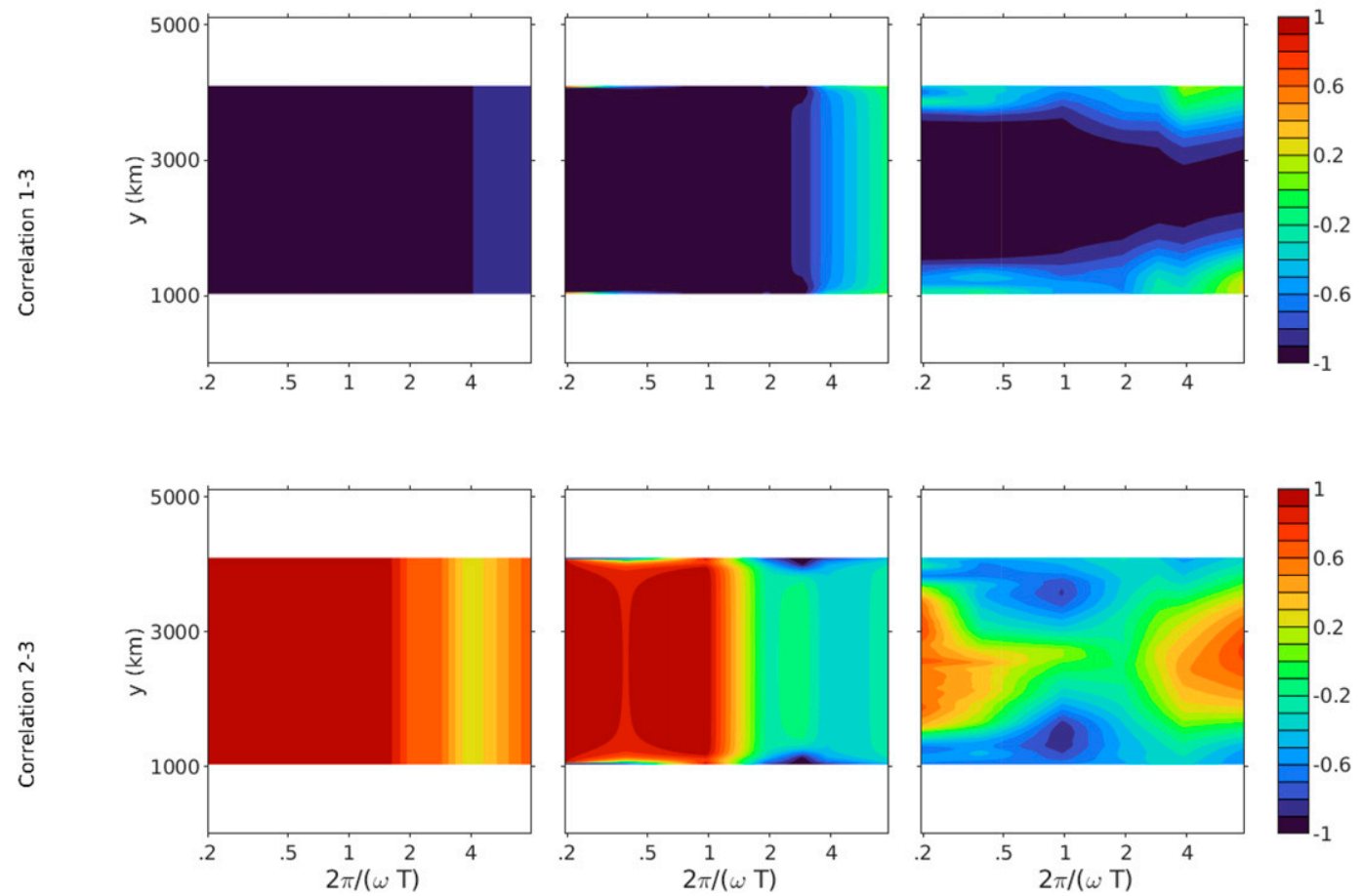

FIG. 11. Correlation between layers (top) 2 and 1, (middle) 3 and 1, and (bottom) 3 and 2 for the (left) theory, (center) linear model, and (right) nonlinear model.

forcing period. The correlation between the MOC at each latitude and the MOC at the central latitude of the subtropical gyre is plotted for the nonlinear QG model runs. The linear model and theory both find that the MOC is highly correlated at all latitudes, so only the nonlinear model results are shown here. For the cases with oscillating wind strength, the upper and deep layers are correlated across both gyres at all frequencies. At high frequencies the correlation is limited to the latitude range of the wind stress curl, while at low frequencies the correlation extends beyond the limits of the wind stress due to the meridional eddy fluxes. However, the middle layer shows a loss of correlation at periods less than the basin crossing time scale, especially so near the gyre boundary. This is due to baroclinic instability at the gyre boundary. The eddy fluxes in the middle layer oppose the Ekman-driven overturning and so the amplitude of the MOC is greatly reduced (see also Fig. 7) and the correlation breaks down.

The meridional coherence for the cases with an oscillating latitude of the wind stress is anticorrelated between gyres, as expected (Fig. 12, right side). The influence of the eddy-driven MOC is evident at high and low latitudes for long forcing periods. There is some loss of coherence between gyres in the middle layer at shorter periods but it is not as strong as for the cases with varying wind strength.

\section{e. More realistic forcing}

The theory and numerical examples in the preceding sections provide a useful exposition of the dynamics of the wind-forced MOC as a function of forcing frequency. However, they were cases in which the forcing is periodic in time and of a single frequency, while for the real ocean the 

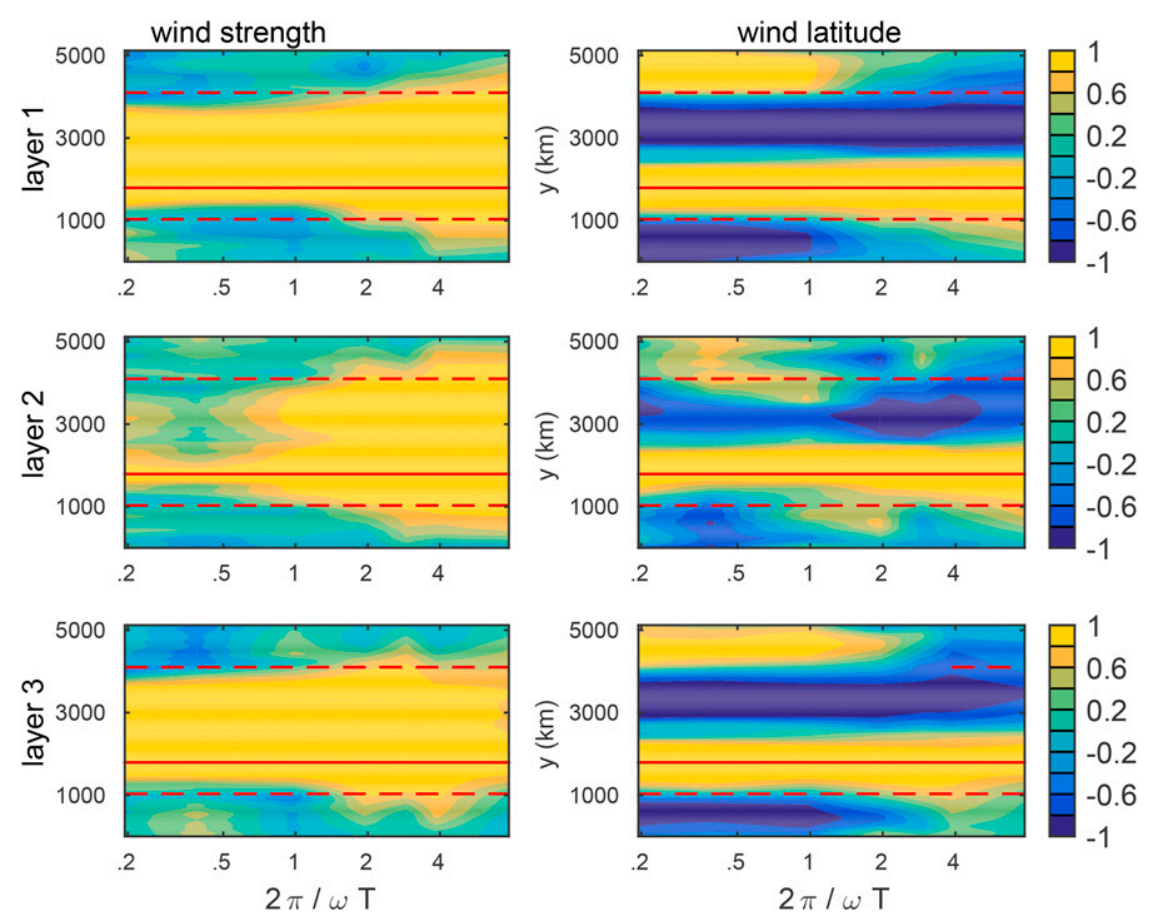

FIG. 12. Correlation of the MOC with that at the central latitude of the subtropical gyre (solid red line) as a function of latitude and forcing period for (left) variations in wind strength and (right) variations in wind latitude. Dashed red lines mark the limits of direct wind forcing.

forcing is not periodic and contains energy at all frequencies. If the responses to forcing by different frequency components are added linearly, one might be able to predict the response to more complex forcing scenarios. Perhaps more problematic is that realistic forcing, particularly for interannual and longer periods, is not periodic in time. For example, the strong reduction in the MOC at the RAPID-MOCHA array in 2009/10 was partly caused by an anomalous wind event in that year alone (McCarthy et al. 2012). To test the applicability of the theory to more realistic situations, the nonlinear QG model was spun up for 20 years with a constant wind stress and then run for an additional 20 years with a wind stress that varies according to

$$
\tau=\tau_{0}\left(1+a_{1} \sin \omega_{1} t+a_{2} \sin \omega_{2} t+a_{3} \sin \omega_{3} t\right),
$$

where $a_{1}=0.25, a_{2}=0.2$, and $a_{3}=0.15$, and the forcing frequencies are $\omega_{1}=2 \pi /(1 \mathrm{yr}), \omega_{2}=2 \pi /(5 \mathrm{yr})$, and $\omega_{3}=2 \pi /(20 \mathrm{yr})$. This provides a strong seasonal signal but also variability at interannual and decadal periods. While the forcing is still periodic in nature, the analysis is applied to the time period immediately after spinup, so the basin response is to an abrupt onset of time-dependent forcing. The resulting MOC at the midlatitude of the basin (with a 90-day smoothing to suppress mesoscale noise) is shown in Fig. 13. The initial spinup of the subtropical and subpolar gyres takes place over the first 10 years of integration with internal variability emerging after several years. The inherent variability with constant wind stress is less than $1 \mathrm{~Sv}$. After 20 years the time-dependent forcing starts and the MOC variability increases to $O(4 \mathrm{~Sv})$. The signal is dominated by the annual cycle but there is also variability on 5- and 20-yr time scales.

The theory in section 4 was applied to this forcing scenario by calculating the analytic response to each of the timedependent components of the wind stress and linearly adding the predicted MOC for each. The resulting MOC for each layer is shown in Fig. 13 by the dashed lines. The general fluctuations found in the model are predicted by the theory, although there are differences. The theory tends to overpredict the magnitude of the response, consistent with Fig. 7, but is of the right order of magnitude and the phase is well represented. The correlation between the theory and numerical result is $0.80,0.18$, and 0.83 for the upper, mid-, and deep layers. The RMS difference between the nonlinear model MOC and the theory is 1.4, 0.6, and 1.0 Sv for the upper, mid-, and deep layers. The RMS variability of the MOC in the nonlinear model between years 20 and 40 is $1.7,0.401 .5 \mathrm{~Sv}$ for the same three layers. It is noteworthy that the agreement between the theory and model in the first two years of time-dependent forcing is very good, indicating that the theory remains relevant even when the initial conditions do not have the memory of previous periodic forcing cycles.

The theory has some predictive skill for the upper and deep layers (high correlation; reduced RMS difference) but it has no skill for the middle layer (low correlation; increased RMS difference). The disagreement between model and theory for the middepth MOC is at least partly caused by baroclinic instability and the related eddy fluxes, as outlined in the previous sections. The correlation between the linear model and the theory increases to $0.95,0.42$, and 0.87 for the three layers. 
a)
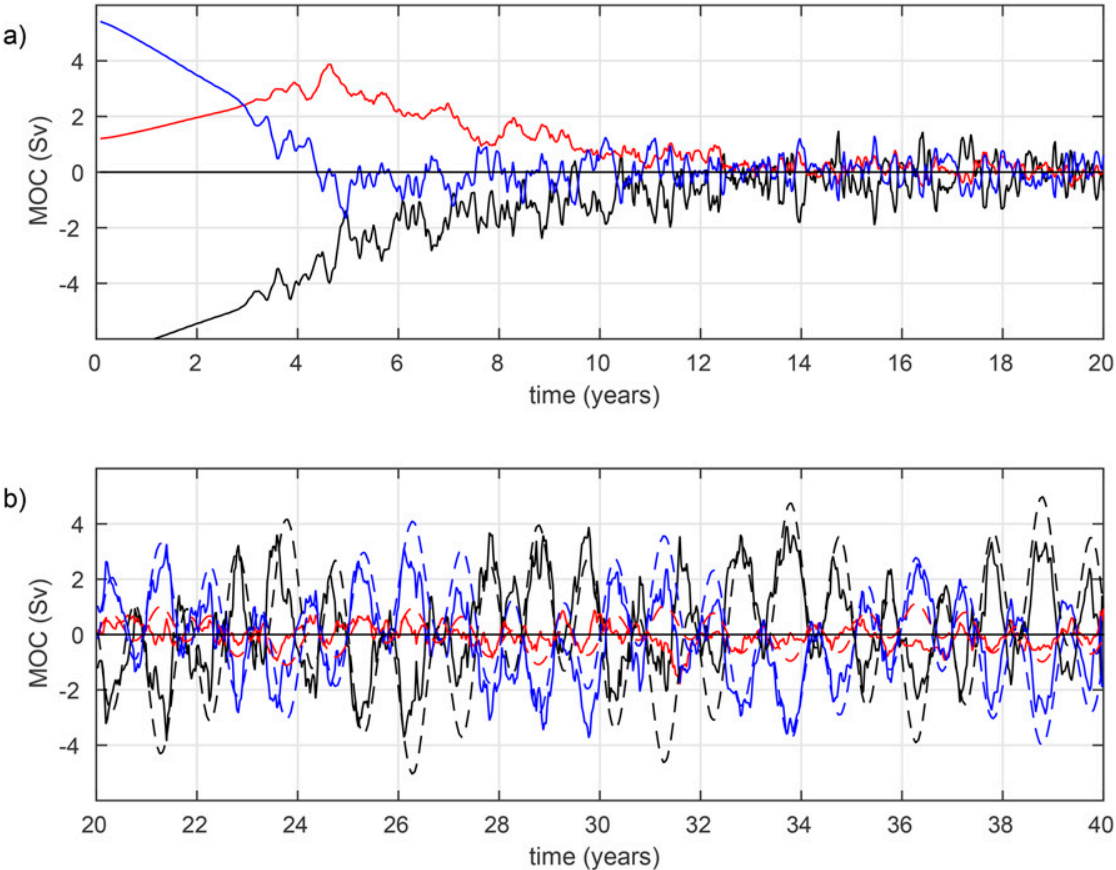

FIG. 13. Meridional overturning circulation for a case with simultaneous forcing frequencies of 1, 5, and 20 years from (25). The time-dependent winds are turned on at year 20. (a) Spinup years 0-20; (b) forced years 20-40. Solid lines are from the nonlinear model; dashed lines are from the linear theory. Black, layer 1; red, layer 2; and blue, layer 3.

So, while there are differences between the theory and nonlinear model the salient point is that the theory does a good job of predicting the MOC response even though the theory was applied to the time period immediately after the time-dependent forcing was turned on and was subject to variations on annual to decadal time scales. This indicates that the MOC response to variable forcing is fast, making the periodic analysis useful even for time periods as short as the forcing period.

\section{Discussion and conclusions}

Recent observations and basin-scale modeling studies have begun to construct a three-dimensional picture of the MOC and its variability. At seasonal to interannual time scales, wind forcing has been found to be the dominant forcing mechanism while buoyancy forcing is more important at longer time scales. Wind variability gives rise to low-frequency variability in heat storage below the mixed layer and in air-sea exchange at the surface. While the importance of wind forcing has been diagnosed in observations, reanalysis, and forward numerical models, the theoretical understanding of the basic mechanisms of wind-forced MOC variability is incomplete. The simplest connection between wind and MOC is based on meridional Ekman transport forcing a barotropic return flow, which represents both a meridional circulation and, because the ocean is stratified, a meridional heat transport. However, observations clearly indicate that the wind-driven MOC is both baroclinic and nonlocal, so this framework is insufficient.
The present study combines an analytic quasigeostrophic theory and an idealized configuration of a three-layer quasigeostrophic numerical model to understand the threedimensional structure of the midlatitude wind-driven MOC variability. Two types of wind variability are considered: changes in wind strength and changes in wind latitude. This is consistent with the recent study by Zou et al. (2020), who found that the wind stress variability in the North Atlantic is dominated by two modes, one representing a change in wind strength and one representing a shifting of the wind stress pattern with latitude. The theory and linear model show that changes in wind strength drive an MOC that connects the subtropical and subpolar gyres. At short forcing periods (seasonal to interannual) the linear response is largely barotropic and independent of forcing frequency. However, for forcing periods on the order of the first-mode baroclinic Rossby wave time scale the MOC is enhanced above that expected by the Ekman transport alone as a result of a phase shift between the first and second baroclinic modes. At even longer period forcing the passing of the first baroclinic mode and the prominence of the second baroclinic mode causes the MOC to decrease in the deep ocean and increase at middepths. Higher baroclinic modes were also inferred to be important for MOC variability in an ECCO state estimate by Tandon et al. (2020). A similar frequency dependence is found for variations in the wind latitude but for these cases the overturning is largely isolated within each gyre and anticorrelated between the subtropical and subplolar gyres. This pattern of cross-gyre MOC for variations in wind strength and anticorrelation 
between gyres for variations in wind latitude are consistent with the findings of Zou et al. (2020) based on realistic numerical models and reanalysis products. High-frequency, localized wind anomalies, inherent internal variability or a mixture of changes in wind strength and location can result in a loss of meridional coherence in the MOC, as found by Bingham et al. (2007).

Nonlinearities and mesoscale eddies alter the MOC expected from linear dynamics in two important ways. First, baroclinic instability drives an overturning cell at middepth that opposes the linear wind-driven overturning. This causes a smaller-amplitude MOC at middepths and a stronger overturning in the deep ocean. These eddy fluxes also alter the phase of the middepth overturning such that at seasonal to interannual time scale the middepth MOC is more in phase with the Ekman transport while the linear theory predicts that it would be $180^{\circ}$ out of phase. The second important role of mesoscale eddies is to extend the MOC outside the latitude range of direct wind forcing. This results from baroclinic instability responding to the fluctuating strengths of the winddriven subpolar and subtropical gyres. Mesoscale eddies cause a loss of correlation at middepths between local wind forcing and the MOC, and between the MOC in the subtropical and subpolar gyres, especially at seasonal to interannual time scales. This weak correlation is consistent with recent observations (Frajka-Williams et al. 2016) and numerical model results (Zou et al. 2019).

These findings indicate that the response of the MOC to variable winds depends strongly on the mode of variability in the wind, the frequency of forcing, and the latitude. Higher baroclinic modes are important for both the strength and phase of the MOC at midlatitudes for interannual to decadal time scales. Mesoscale eddies can also alter the strength and phase of the MOC, particularly at middepths. These results are consistent with, and may provide simple explanations for, recent descriptions of the three-dimensional MOC. The idealized quasigeostrophic approach adopted here provides some conceptual and analytical advantages, but neglects some potentially important physical processes, including: large isopycnal displacements, diapycnal mixing and surface heat flux, spatially variable deformation radius and stratification, boundary waves, bottom topography, and a mean meridional overturning circulation. The theoretical framework provided here should provide a foundation for analysis of more complete circulation models and observations.

Acknowledgments. This study was supported by National Science Foundation Grant OCE-1947290. Doctors Pavel Berloff and Igor Shevchenko are thanked for providing the PEQUOD QG numerical model and answering numerous questions about its formulation. Conversations with Dr. Joseph Pedlosky were helpful for derivation of the analytic solutions in section 4. Comments and suggestions from two anonymous reviewers helped to improve the presentation.

\section{REFERENCES}

Abraham, J. P., and Coauthors, 2013: A review of global ocean temperature observations: Implications for ocean heat content estimate and climate change. Rev. Geophys., 51, 450-483, https://doi.org/10.1002/rog.20022.

Anderson, D. L. T., and A. Gill, 1975: Spin-up of a stratified ocean with application to upwelling. Deep-Sea Res., 22, 583-596, https://doi.org/10.1016/0011-7471(75)90046-7.

Berloff, P., 2016: Dynamically consistent parameterization of mesoscale eddies-part II: Eddy fluxes and diffusivity from transient impulses. Fluids, 1, 22, https://doi.org/10.3390/ fluids1030022.

- 2018: Dynamically consistent parameterization of mesoscale eddies. Part III: Deterministic approach. Ocean Modell., 127, 1-15, https://doi.org/10.1016/j.ocemod.2018.04.009.

Biastoch, A., C. W. Böning, J. Getzlaff, J. M. Molines, and G. Madec, 2008: Causes of interannual-decadal variability in the meridional overturning circulation of the midlatitude North Atlantic Ocean. J. Climate, 21, 6599-6615, https://doi.org/ 10.1175/2008JCLI2404.1.

Bingham, R. J., C. W. Hughes, V. Roussenov, and R. G. Williams, 2007: Meridional coherence of the North Atlantic meridional overturning circulation. Geophys. Res. Lett., 34, L23606, https://doi.org/10.1029/2007GL031731.

Bryan, K., 1982: Seasonal variation in meridional overturning and poleward heat transport in the Atlantic and Pacific Oceans. J. Mar. Res., 40, 39-53.

Buckley, M. W., R. M. Ponte, G. Foget, and P. Heimbach, 2015: Determining the origins of advective heat transport convergence variability in the North Atlantic. J. Climate, 28, 39433956, https://doi.org/10.1175/JCLI-D-14-00579.1.

Evans, D. G., J. Toole, G. Forget, J. D. Zika, A. C. N. Garabato, A. J. Nurser, and L. Yu, 2017: Recent wind-driven variability in Atlantic water mass distribution and meridional overturning circulation. J. Phys. Oceanogr., 47, 633-647, https:// doi.org/10.1175/JPO-D-16-0089.1.

Frajka-Williams, E., and Coauthors, 2016: Compensation between meridional flow components of the Atlantic MOC at $26^{\circ} \mathrm{N}$. Ocean Sci., 12, 481-493, https://doi.org/10.5194/os-12-4812016.

Grist, J. P., and Coauthors, 2010: The roles of surface heat flux and ocean heat transport convergence in determining Atlantic Ocean temperature variability. J. Phys. Oceanogr., 60, 771790, https://doi.org/10.1007/s10236-010-0292-4.

Häkkinen, S., P. B. Rhines, and D. L. Worthen, 2015: Heat content variability in the North Atlantic Ocean in ocean reanalysis. Geophys. Res. Lett., 42, 2901-2909, https://doi.org/10.1002/ 2015GL063299.

Huang, R. X., 2015: Heaving modes in the world oceans. Climate Dyn., 45, 3563-3591, https://doi.org/10.1007/s00382-0152557-6.

Jayne, S. R., and J. Marotzke, 2001: The dynamics of ocean heat transport variability. Rev. Geophys., 39, 385-411, https:// doi.org/10.1029/2000RG000084.

Kanzow, T., and Coauthors, 2010: Seasonal variability of the Atlantic meridional overturning circulation at $26.5^{\circ} \mathrm{N}$. J. Climate, 23, 5678-5698, https://doi.org/10.1175/2010JCLI3389.1.

Karabasov, S. A., P. S. Berloff, and V. M. Goloviznin, 2009: CABARET in the ocean gyres. Ocean Modell., 30, 155-168, https://doi.org/10.1016/j.ocemod.2009.06.009.

Knight, J. R., R. J. Allan, C. K. Folland, M. Vellinga, and M. E. Mann, 2005: A signature of persistent natural thermohaline circulation cycles in observed climate. Geophys. Res. Lett., 32, L20708, https://doi.org/10.1029/2005GL024233.

Latif, M., C. Böning, J. Willebrand, A. Biastoch, J. Dengg, N. Keenlyside, G. Madec, and U. Schweckendiek, 2006: Is the 
thermohaline circulation changing? J. Climate, 19, 4631-4637, https://doi.org/10.1175/JCLI3876.1.

Levitus, S., and Coauthors, 2012: World ocean heat content and thermosteric sea level change (0-2000 m), 1955-2010. Geophys. Res. Lett., 39, L10603, https://doi.org/10.1029/ 2012GL051106.

Lozier, M. S., S. Leadbetter, R. G. Williams, V. Roussenov, M. S. C. Reed, and N. J. Moore, 2008: The spatial pattern and mechanisms of heat-content change in the North Atlantic. Science, 319, 800-803, https://doi.org/10.1126/science.1146436.

McCarthy, G., and Coauthors, 2012: Observed interannual variability of the Atlantic meridional overturning circulation at $26.5^{\circ}$ N. Geophys. Res. Lett., 39, L19609, https://doi.org/10.1029/ 2012GL052933.

McCarthy, G. D., and Coauthors, 2015: Measuring the Atlantic meridional overturning circulation at $26.5^{\circ} \mathrm{N}$. Prog. Oceanogr. 130, 91-111, https://doi.org/10.1016/j.pocean.2014.10.006.

Pillar, H., P. Heimbach, H. Johnson, and D. Marshall, 2016: Dynamical attribution of recent variability in Atlantic overturning. J. Climate, 29, 3339-3352, https://doi.org/10.1175/ JCLI-D-15-0727.1.

Polo, I., J. Robson, R. Sutton, and M. A. Balmaseda, 2014: The importance of wind and buoyancy forcing for the boundary density variations and the geostrophic component of the AMOC at $26^{\circ}$ N. J. Phys. Oceanogr., 44, 2387-2408, https:// doi.org/10.1175/JPO-D-13-0264.1.

Roemmich, D., and C. Wunsch, 1985: Two transatlantic sections: Meridional circulation and heat flux in the subtropical North Atlantic Ocean. Deep-Sea Res., 32, 619-664, https://doi.org/ 10.1016/0198-0149(85)90070-6.

Spall, M. A., and D. Nieves, 2020: Wind-forced variability of the remote overturning circulation. J. Phys. Oceanogr., 50, 455469, https://doi.org/10.1175/JPO-D-19-0190.1.

Tandon, N. F., O. A. Saenko, M. A. Kane, and P. J. Kushner, 2020: Interannual variability of the global meridional overturning dominated by the Pacific Ocean. J. Phys. Oceanogr., 3, 559574, https://doi.org/10.1175/JPO-D-19-0129.1.

White, W. B., 1977: Annual forcing of baroclinic long waves in the tropical North Pacific Ocean. J. Phys. Oceanogr., 7, 50-61, https://doi.org/10.1175/1520-0485(1977)007<0050:AFOBLW $>$ 2.0.CO;2.

Williams, R. G., V. Roussenov, D. Smith, and M. S. Lozier, 2014: Decadal evolution of ocean thermal anomalies in the North Atlantic: The effects of Ekman, overturning, and horizontal transport. J. Climate, 27, 698-719, https://doi.org/10.1175/ JCLI-D-12-00234.1.

Yang, J., 2015: Local and remote wind-stress forcing of the seasonal variability of the Atlantic Meridional Overturning Circulation (AMOC) transport at $26.5^{\circ}$ N. J. Geophys. Res., 120, 24882503, https://doi.org/10.1002/2014JC010317.

Yeager, S., and G. Danabasoglu, 2014: The origins of late twentieth-century variations in the large-scale North Atlantic circulation. J. Climate, 27, 3222-3247, https://doi.org/10.1175/ JCLI-D-13-00125.1.

Zhao, J., and W. Johns, 2014a: Wind-driven seasonal cycle of the Atlantic meridional overturning circulation. J. Phys. Oceanogr., 44, 1541-1562, https://doi.org/10.1175/JPO-D-130144.1.

—_ and —_, 2014b: Wind-forced interannual variability of the Atlantic Meridional Overturning Circulation at $26.5^{\circ} \mathrm{N}$. J. Geophys. Res., 119, 2403-2419, https://doi.org/10.1002/ 2013JC009407.

Zou, S., M. S. Lozier, and M. Buckley, 2019: How is meridional coherence maintained in the lower limb of the Atlantic meridional overturning circulation? Geophys. Res. Lett., 46, 244252, https://doi.org/10.1029/2018GL080958.

,-- , and X. Xu, 2020: Latitudinal structure of the meridional overturning circulation variability on interannual to decadal time scales in the North Atlantic Ocean. J. Climate, 33, 38453862, https://doi.org/10.1175/JCLI-D-19-0215.1. 